\section{Indizien und Indikatoren zur Umsetzung des Vorsorgeprinzips}

\author{
von Arnim von Gleich, Christian Pade und \\ Henning Wigger, Universität Bremen
}

Handeln mit unzureichendem Wissen bestimmt unseren Alltag. Es gibt also eine ganze Reihe von Gründen, den Fokus in der TA-Forschung auf bloße Indizien zu legen. Dabei geht es zunächst v. a. um die politisch-praktische Umsetzung eines wirksamen Vorsorgeprinzips, zumindest für solche gesellschaftlichen Realexperimente, bei denen beim ersten erkennbaren Auftreten negativer Konsequenzen kaum noch korrigierend eingegriffen werden kann. Die Beschäftigung mit Indizien ist zudem relevant für die methodische Weiterentwicklung der Technikfolgenabschätzung und ihre epistemologische Selbstvergewisserung.

Aufbauend auf Erfahrungen aus den Bereichen Chemikalien, Gentechnik, Nanotechnologien und Synthetische Biologie wird in diesem Text die These vertreten, dass eine bewusste und begründete Zurücknahme der Evidenzanforderungen an „Gründe für Besorgnis“ als Auslöser für Maßnahmen nach dem Vorsorgeprinzip - von wissenschaftlichen Beweisen eines Risikos zu bloßen Indizien einer Gefährdung - einen notwendigen, längst überfälligen aber auch in bestimmten Bereichen schon durchaus praktizierten Schritt darstellt.

\section{Einleitung}

Die Vorstellung, dass wir über alle Wirkungen aller Innovationen immer schon im Vorhinein Bescheid wissen sollen, überschätzt unsere Erkenntnismöglichkeiten, würde zu viele Ressourcen binden und Innovationsprozesse weitgehend blockieren. Die Grenzen des bisher vorherrschenden Vorgehens nach dem Prinzip Versuch und Irrtum liegen spätestens dort, wo wir nach dem Auftreten von Problemen nicht mehr korrigierend eingreifen können. Der Imperativ der Technikfolgenabschätzung sollte demzufolge nicht lauten: „Handle erst, wenn du alle Folgen deines Handelns überschauen kannst“, sondern: „Handle so (bzw. stelle sicher), dass danach jederzeit noch korrigierend eingegriffen werden kann".

Hochgeschraubte Forderungen nach wissenschaftlichen Evidenzen und Beweisen sind jedoch ungebrochen gefragt im Diskurs über Technikfolgen. Hierin treffen sich die Argumente der sog. „Betreiber“ (z. B. mit Blick auf die geforderten wissenschaftlichen Standards toxikologischer Studien') mit den sog. „Bedenkenträgern“ (,die Wirkungen sind noch völlig unbekannt bzw. unerforscht", „,no data, no market" ${ }^{\text {(2) }}$ ) und nicht zuletzt mit der wissenschaftlichen Binnenorientierung der TA-Community (wer möchte schon „unsichere“ Ergebnisse produzieren?). Tendenziell unerfüllbare Evidenzforderungen werden in Auseinandersetzungen zwischen „Betreibern“ und „Bedenkenträgern“ gerne wie eine heiße Kartoffel hin- und hergeworfen (,beweise doch, dass es gefährlich bzw. ungefährlich ist"). Dieses Spiel ist zwar sehr beliebt, es trägt aber zu einer verantwortungsvollen und vorsorgeorientierten Innovationspraxis wenig bei.

Auch Indizien ${ }^{3}$ beruhen in der Regel auf Fakten. Ihre Überprüfung sollte - insbesondere, wenn es um Besorgnisgründe geht - mit überschaubarem Aufwand zu einem hinreichend evidenten Ergebnis führen. Die Unsicherheit bezieht sich dann allerdings v. a. darauf, dass diese Fakten als Indikatoren gewertet werden, als Verweis auf etwas weiter reichendes, als Ausgangspunkte für Vermutungen über Schuld bzw. Unschuld im Gerichtsverfahren oder als „Anlass für Besorgnis“ in der Umweltpolitik und Technikfolgenabschätzung ${ }^{4}$. Die Unsicherheit bezieht sich hier also „nur“ auf die Fähigkeit zur Indikation. Noch schwieriger wird es, wenn auch die Überprüfbarkeit des Vorliegens oder Nicht-Vorliegens von Indizien unsicher ist $^{5}$. Angesichts von Unsicherheiten, die die in vielen Gesetzen geforderte „Evidenz des Vorliegens einer Gefahr oder eines Risikos" angesichts von Wissenslücken nicht zulassen, können und müssen bei der Umsetzung des Vorsorgeprinzips auch schon bestimmte „Anlässe für Besorgnis“" (reasonable concerns) ausreichen, um Vorsorgemaßnahmen zu begründen. Jedoch schließt sich dann die spannende politisch-praktische Frage nach dem Verhältnis des Evidenzgrades der Indikation für Vorsorgemaßnahmen zu deren gesellschaftlicher Eingriffstiefe an. Mit welchem Evidenzgrad 
lassen sich besonders tiefgreifende Vorsorgemaßnahmen, wie z. B. ein Moratorium, begründen?

Das Problem hat im Grunde zwei Seiten. So lässt sich allein mit der Tatsache, dass man über mögliche Folgen einer Technik, eines Stoffes oder Eingriffs wenig weiß, eine völlige Unterlassung oder ein Moratorium wohl kaum hinreichend begründen. Es ist durchaus klug, vor einem weitreichenden Eingriff in die Freiheitsrechte von Akteuren auf überprüfbare Indizien zu pochen. Die Unterlassung bzw. Zurückstellung potenziell weitreichender gesellschaftlicher Realexperimente kann dann allerdings mit Angabe guter Besorgnisgründe durchaus legitimiert werden. Doch oft kennt man diese Unterlassungsgründe nicht im Vorhinein (vgl. die Fülle von Beispielen angefangen von DDT über FCKW bis hin zu hormonähnlichen Wirkungen von Industriechemikalien; vgl. EEA 2001 und 2013). Mit Blick auf dieses kritische Wissensverhältnis (das die Form des Collingridge-Dilemmas aufweist $\left.{ }^{\circ}\right)$, ist die Erarbeitung von Indizien insbesondere für Technologien, Stoffe und Eingriffe wichtig, bei denen, wenn sich problematische Wirkungen zeigen, nicht mehr adäquat korrigierend eingegriffen werden kann. Die Lösung dieses Problems gehört zu den zentralen wissenschaftlichen Aufgaben zur Begründung und Unterstützung der politischen Umsetzung eines notfalls tiefgreifenden Vorsorgeprinzips, das, dort wo dies begründet und notwendig ist, über allzeit angebrachte Behutsamkeits- und Vorsichtsmaßnahmen weit hinausreicht.

Akteure, welche den Stellenwert von Indizien in der Technikfolgenabschätzung steigern möchten und an der Bestimmung und Verbesserung ihrer (epistemischen) Qualität arbeiten etwa nach dem Vorbild der Rechtsprechung, in der Indizien schon immer eine wichtige Rolle spielten - sind allerdings dünn gesät ${ }^{7}$. Dies ist umso bemerkenswerter, als Handeln unter Unsicherheit und Entscheidungen auf der Basis von mehr oder minder expliziten Indizien, genau besehen, die alltägliche Praxis darstellt. Erstaunlicherweise fehlt eine entsprechende wissenschaftliche Aufarbeitung selbst dort, wo - wie z. B. in der europäischen Chemikaliengesetzgebung REACH - die Politik längst aufgrund von bloßen Indizien recht weitreichende Vorsorgemaßnahmen vorschreibt ${ }^{8}$.

\section{Fokus auf Indizien}

Es gibt eine ganze Reihe von Gründen, den Fokus in der TA-Forschung auf Indizien zu legen.

Hier sollen drei dieser Gründe herausgegriffen werden:

a) Pragmatik und Effizienz: Das Aufwand-Ertragsverhältnis bei der Überwindung des NochNicht-Wissens (dealing with known unknowns) Insbesondere im Bereich Chemikalien und Nanomaterialien ist seit geraumer Zeit klar, dass es schlicht unmöglich ist, allumfassende Testergebnisse zur Voraussetzung für das InVerkehr-bringen von (neuen) Stoffen zu machen $^{9}$. Die ungeheure Vielfalt der Substanzen und ihrer Erscheinungsformen sowie die durch den globalen Wettbewerb hochgetriebene Innovationsgeschwindigkeit stehen dem entgegen. Die Europäische Chemikaliengesetzgebung $\mathrm{REACH}{ }^{10}$ reagierte auf diese Situation in mehrfacher Hinsicht intelligent und pragmatisch, indem sie die Wissens- bzw. Testanforderungen zu möglichen problematischen Neben- und Folgewirkungen von Stoffen zum einen an die in Verkehr gebrachten Mengen und zum anderen an bestimmte besonders Besorgnis erregende Stoff-Qualitäten knüpfte ${ }^{11}$. Beide Indizien für Expositions- und Gefährdungspotenziale beziehen sich auf gut überprüfbare Tatbestände (Mengen und Stoffqualitäten), die schon bestimmte Vorsorgemaßnahmen auslösen, obwohl zum „Beweis“ der Existenz von Gefahren und Risiken zusätzliche, derzeit nicht verfügbare, Informationen über Gefährdungen und Expositionen nötig wären.

REACH nimmt in seiner pragmatischen, indizienbasierten Regelungspraxis vor dem Hintergrund der Zwickmühle zwischen Untersuchungsaufwand und Innovationsgeschwindigkeit also durchaus große Wissenslücken in Kauf. Man kann das In-Verkehr-bringen unzureichend geprüfter Stoffe mit guten Argumenten für unverantwortlich halten. Doch was heißt genau unzureichend? Wie viele Tests sollen vorgeschrieben werden? Wie viel müssen wir definitiv vor dem In-Verkehr-bringen wissen? Mehr Folge-Wissen mit etwas mehr TA-Aufwand ist im Prinzip immer zu begrüßen. Wenn man aber dazu tendiert, diese Wis- 
sensanforderungen beliebig hoch zu schrauben, sieht man sich über kurz oder lang mit der Frage konfrontiert, welchen Anteil nicht nur an finanziellen Ressourcen - man denke an die ethische Problematik der Tierversuche - eine Gesellschaft für diese Form der Vorsorge aufwenden möchte. Eine entsprechende Diskussion findet bisher noch nicht statt. Wir kennen jenseits der Anzahl von Tierversuchen kaum die aktuellen Zahlen, und es stehen auch keine Vorschläge im Raum, welches finanzielle Niveau anstrebenswert wäre ${ }^{12}$. Wären eher 2 oder $10 \%$ des BIP angemessen für Risikoforschung und Risikomanagement oder gar $30 \%$ der Aufwendungen für Innovationen?

b) Unvorhersehbarkeiten und Nicht-Wissbarkeiten (dealing with unknown unknowns and unknowables)

Die Zukunft ist prinzipiell offen. Früh im Innovationsprozess sind die Anwendungskontexte, die Anwendungsintentionen (inkl. Missbrauchsmöglichkeiten) von Technologien und damit auch mögliche Expositionen gegenüber Gefährdungspotenzialen noch nicht bekannt. Ein gangbarer Weg zur Auslotung dieser Ungewissheiten sind Anwendungsszenarien, die REACH auch von den Anbietern neuer Stoffe einfordert. Diese Szenarien müssen mit Annahmen arbeiten, welche sich wiederum auf bisherige Erfahrungen und zukunftsorientierte Indizien stützen müssen.

Eine weitere wichtige Quelle von Ungewissheit liegt in der Komplexität der Systeme, in welche eingegriffen wird. Hier ist mit Nicht-Linearitäten zu rechnen, Prognosen werden unmöglich. Die Forderung, aus diesen Gründen prinzipiell die Finger von komplexen Systemen $\mathrm{zu}$ lassen, ist nicht praktikabel. Zumindest, wenn wir als Kennzeichen von komplexen Systemen die Zahl und Diversität ihrer Elemente sowie die Zahl und Qualität der Verknüpfungen zwischen diesen (inkl. Rückkopplungsmechanismen) verstehen, müssen wir wohl davon ausgehen, dass wir permanent und allenthalben in komplexe Systeme eingreifen. Wobei sich in der Regel zeigt, dass die meisten dieser Systeme vergleichsweise träge und gutmütig reagieren. Das prinzipiell mögliche explosionsartige „Abrauschen“, die Bifurkation auf ein völlig neues Trajekt oder ein völliger Zusammenbruch (je nach Sichtweise auch bloß ein Switchen in einen anderen Stabilitätszustand) sind eher die Ausnahme als die Regel. Aber derartige Reaktionen sind durchaus möglich. In dieser Situation kommt es darauf an, vor dem Eingriff „Indizien“ über die Vulnerabilität bzw. Resilienz des Systems ${ }^{13}$ zu gewinnen, über seinen aktuellen Zustand, sein aktuelles Trajekt sowie über seine Reaktionsfähigkeiten, insbesondere seine Widerstands-, Anpassungs-, Improvisations- und Innovationsfähigkeiten (vgl. Abb. 1).

c) Fokussierung auf das Wesentliche, die Erhaltung von Handlungsspielräumen: Korrigierbarkeit, Rückholbarkeit, Fehlerfreundlichkeit Nicht mehr korrigierbare Konsequenzen von Eingriffen in Systeme können im Prinzip auf drei Wegen entstehen: 1. als kumulative Wirkung unzähliger je für sich vergleichsweise

Abb. 1: Indizien und Indikatoren als Wissensquellen für eine vorsorgeorientierte Innovationspolitik

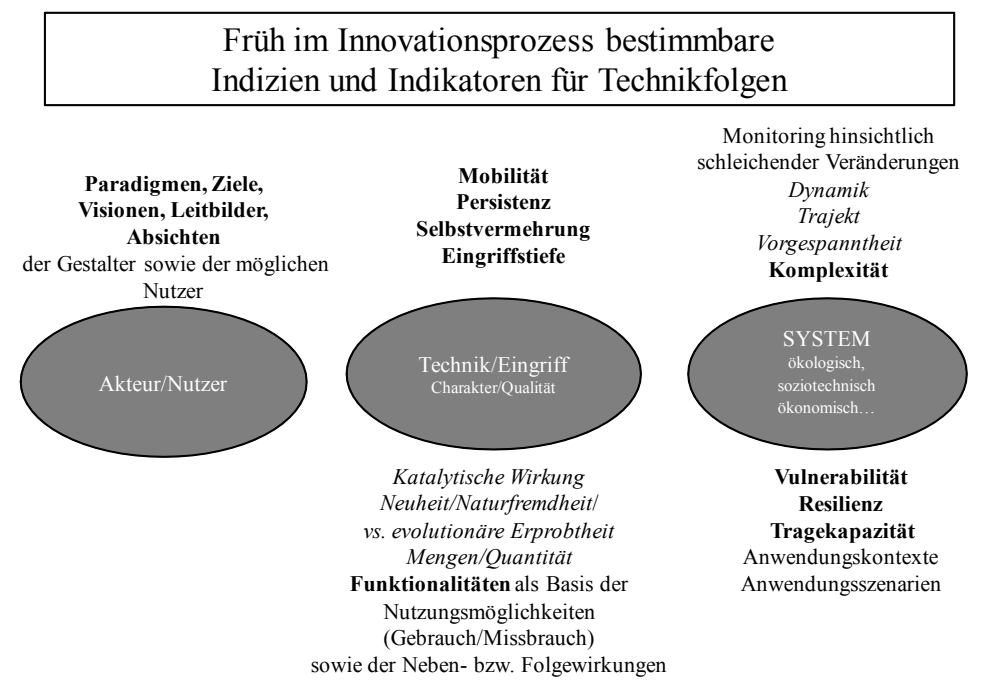

Quelle: Eigene Darstellung 
harmloser Eingriffe (Eingriffsquantitäten), 2. als Folge eines vergleichsweise harmlosen Eingriffs in ein besonders labiles und vulnerables System (Systemzustand), 3. als Folge eines besonders tiefgreifenden und wirkmächtigen Eingriffs in ein ansonsten vergleichsweise gutmütiges System (Eingriffsqualitäten). Damit wird der Fokus in Ergänzung des eben angesprochenen „Zustands“ des Systems, in das eingegriffen wird, auf den „Charakter des Eingriffs“ gelegt, auf seine Qualitäten aber auch auf seine Quantitäten (vgl. Abb. 1).

Als besonders interessant im Sinne der Umsetzung des Vorsorgeprinzips ist die qualitative REACH-Regelung zu werten, dass für Stoffe, die in der Umwelt und in Organismen nicht abgebaut werden und sich dort anreichern können (very persistent and very bioaccumulative substances), ein Zulassungsverfahren zwingend vorgeschrieben wird. Dies gilt sozusagen ,schadensunabhängig“" selbst dann, wenn noch gar keine Wirkungsmodelle bzw. Vorstellungen über mögliche Schadensbilder vorliegen. Allein diese Stoffeigenschaften werden von REACH als Indiz gewertet für eine hohe Exposition und somit für das zweite Element im toxikologischen Risikobegriff (Risiko als Funktion von Gefährdungspotenzial und Exposition). Auf diese Weise wird ganz praktisch - und das ist der entscheidende Punkt - das Nicht-Wissen in das Handlungsmodell eingebaut, um z. B. eine tendenziell globale und irreversible Exposition zu unterbinden. Mit dieser Regelung wurde offenkundig auf die Erfahrungen mit FCKWs und anderen ,persistant organic pollutants“ reagiert ${ }^{14}$. Die Stoffeigenschaften Persistenz, Mobilität und Naturfremdheit erweitern extrem die Möglichkeiten für hohe Expositionen und adverse Wirkungen in den unterschiedlichsten Kontexten. Sie erhöhen die Expositionsmöglichkeiten ins Unermessliche und sind ein Indiz für hohe Eingriffstiefe, NichtRückholbarkeit und geringe Fehlerfreundlichkeit $^{15}$. Bei Eingriffen und Technologien mit derart langen relevanten Wirkungsketten in Raum und Zeit ist ein Vorgehen nach Versuch und Irrtum prinzipiell nicht zu verantworten. REACH schreibt für solche besonders besorgniserregende Stoffe die Suche nach Ersatzstoffen vor. Wenn, um beim Beispiel FCKW zu bleiben, dieselben praktisch-technischen Zwecke durch andere Substanzen wie Propan und Butan erfüllt werden können, die in der Atmosphäre in wenigen Tagen vollständig photochemisch abgebaut werden, ließe sich das Ausmaß des Nicht-Wissens über mögliche Folgen signifikant verringern.

Die Rolle, die bestimmte eingriffstiefe Stoffeigenschaften in der vorsorgeorientierten Chemikalienregulierung spielen, ist übertragbar auf andere Stoffe und Technologien, insbesondere auf Nanomaterialien sowie auf Technologien bzw. Prozesse und Produkte der Gentechnik und Synthetischen Biologie. So kann die Mobilität von Nanopartikeln, insbesondere ihre Fähigkeit, biologische Barrieren (Zellmembranen, Blut-HirnSchranke) zu durchdringen, als Indiz für eine potenziell hohe innere Exposition und Kontamination gewertet werden. Und wenn Produkte der Gentechnik und Synthetischen Biologie die Fähigkeit haben, sich selbst zu vermehren, ist auch dies ein Indiz für eine hohe Exposition und für Probleme bei der Rückholbarkeit. Der Fokus auf derartige „technischen Funktionalitäten" ${ }^{\text {"16 }}$ eröffnet zudem Spielräume für eine vorsorgeorientierte Gestaltung, zumindest dann, wenn die unerwünschte Funktionalität, wie z. B. die Mobilität von Nanomaterialien, sich von der erwünschten technischen Funktionalität analytisch und praktisch trennen lässt. Dies funktioniert natürlich nicht, wenn, wie bei Drug-Delivery-Systemen, ein solches Durchdringen biologischer Barrieren Teil der technischen Funktionalität ist.

Nun weiß man über die Möglichkeiten zur Auslösung derart langer Wirkungsketten nicht in jedem Fall Bescheid. Besonders erschwert wird das Ganze dadurch, dass diese Wirkungen ebenso vom Anwendungskontext und vom Zustand des Systems abhängen, in das eingegriffen wird. Insofern wäre es gut, wenn die Indikatoren für extreme Wirkmächtigkeit nicht nur beim Effekt (also der Ausdehnung der Wirkungsketten in Raum und Zeit) ansetzen würden, sondern bei der Art und Weise, wie diese Entgrenzung hervorgebracht wird. Einen Zugang hierzu bietet das Konzept der Eingriffstiefe. Die Eingriffstiefe, das technische Ansetzen an Strukturen, die die Phänomene sehr weitgehend bestimmen, wird damit zur Quelle einer besonderen Wirkmächtigkeit ${ }^{17}$. 
Zur Umsetzung des Vorsorgeprinzips wäre es somit hilfreich, wenn wir die auslösenden Indizien mit den indizierten erwartbaren Folgen und den ihnen zuordenbaren Risikomanagementmaßnahmen grob in drei Kategorien einteilen könnten:

1. Es muss mit sehr weitreichenden Folgen gerechnet werden, die kaum noch korrigierbar sind. Die Besorgnis ist sehr groß, entsprechende Indizien (insb. Eingriffstiefe und Wirkmächtigkeit der Technologie) begründen sehr weitreichende Vorsorgemaßnahmen (sowohl intensive Folgenforschung als auch eingriffstiefes Risikomanagement).

2. Es muss mit durchaus problematischen, aber räumlich, zeitlich und von der Schwere eingrenzbaren oder ansatzweise reparierbaren Folgen gerechnet werden. Die Besorgnis ist groß, entsprechende Indizien (z. B. akute Toxizität) können sowohl eine weitere Folgenforschung als auch vorsorgeorientierte Maßnahmen im Risikomanagement begründen (z. B. die möglichst weitgehende Vermeidung von Expositionen durch Containment).

3. Problematische Folgen sind möglich, aber aller Voraussicht nach auch rasch korrigierbar. Die Besorgnis ist gering. Die allgemein üblichen Regeln guter Arbeits- bzw. Laborpraxis bzw. der üblichen Hygienestandards reichen zunächst aus. Indizien können weitergehende Vorsichtsund Behutsamkeitsmaßnahmen auslösen.

In Abbildung 1 wurde ein erster Überblick über solche Indizien bzw. Indikatoren versucht. Indizien, die in der ersten Kategorie eine wichtige Rolle spielen sollten, sind dabei fett gedruckt.

\section{Die Besorgnis- und Entlastungskriterien der NanoKommission als Schritt zu einer differenzierten „Evidenzpolitik“}

Nicht nur mit Blick auf die Chemikalienregulierung ist die vorsorgeorientierte Praxis mittlerweile weiter entwickelt als ihre wissenschaftliche Aufarbeitung. Die Regulation, der öffentliche Diskurs und die Stakeholderpartizipation setzen immer früher im Innovationsprozess an. Im Fall der Nanotechnologien wurden die Mitglieder der NanoKommission der Bundesregierung (vertreten waren Bundesministerien, Bundesoberbe- hörden, Landesbehörden, Unternehmen und Verbände, Umwelt- und Verbraucherschutzorganisationen, Kirchen und die Wissenschaft) schon zu einem Zeitpunkt einberufen, zu dem noch kaum nanotechnologische Produkte und Verfahren auf der Basis von Nanomaterialien auf dem Markt waren (2006-2011). Die Kommissionsarbeit verkörperte eine „neue Innovationskultur“, wie es ihr Vorsitzender Wolf Michael Catenhusen formulierte. Als Voraussetzung und Hintergrund für ihren intensiven, auf Kompromisse und Konsens zielenden Diskurs können zwei Aspekte besonders herausgehoben werden: Zum einen die gesellschaftliche Erfahrung, dass zivilgesellschaftliche Akteure mit entsprechender Resonanz in der Öffentlichkeit ganze Technologielinien zu blockieren in der Lage sind, wie das bei der Grünen Gentechnik der Fall war und noch ist. ${ }^{18}$ Zum anderen ein ergebnisoffener Diskurs, weil sich noch keine klaren Positionen zur grundsätzlichen Bewertung dieser Technologielinie verfestigt hatten. Die Mitglieder der NanoKommission konnten sich dadurch in den fünf Jahren ihrer Beratungen in wesentlichen Punkten aufeinander zubewegen. Herauszuhebende Ergebnisse sind ein Leitfaden für Unternehmen zum verantwortungsvollen Umgang mit Nanomaterialien, ein Set von vorläufigen vorsorgeorientierten Bewertungskriterien (Indikatoren!) sowie ein Leitbild für Nachhaltige Nanomaterialien (green nano) ${ }^{19}$.

Als Misserfolge sind hingegen die Tatsachen zu werten, dass der im Konsens erarbeitete Leitfaden zum betrieblichen Umgang mit Nanomaterialien in dieser Form nur von wenigen Unternehmen und Unternehmensverbänden aufgegriffen wurde. Die erarbeiteten Besorgnis- und Entlastungskriterien waren im Bericht der ersten Phase noch mit einer vorläufigen Einstufung nach geringer, mittlerer und hoher Besorgnis verbunden, inklusive entsprechender Managementkonsequenzen. Diese Verknüpfung wurde im Endbericht der zweiten Phase, der eine weiter ausdifferenzierte Fassung der Kriterien enthielt, leider fallen gelassen. Schließlich wurde bis heute von der Bundesregierung keine Initiative gestartet, um entsprechende OECD-Guidelines für ein ,preliminary assessment" auf der Basis solcher Besorgnis- und Entlastungskriterien auszuarbeiten. Ein derartiger Schritt würde ihre nationale und internationale 
Wirksamkeit und Verbreitung maßgeblich befördern. Auch wenn man hinter den ersten beiden unbefriedigenden Umsetzungsaktivitäten handfeste Interessenskonflikte vermuten kann, so ist doch das Ausbleiben der letztgenannten Initiative kaum nachvollziehbar. Ein Blick auf die Kriterien (Abb. 2) zeigt schließlich, bei aller Vorläufigkeit (welche sich etwa im differenten Operationalisierungsgrad der einzelnen Kriterien ausdrückt), dass die meisten der geforderten Informationen für ein ,preliminary assessment“ mit überschaubarem Aufwand zu beschaffen sind. Dies gilt insbesondere für diejenigen Kriterien, die sich auf physikalisch-chemische Eigenschaften der Nanomaterialien beziehen. Danach kann man sich ja immer noch über die Maßnahmen streiten oder verständigen, die ggf. daraus folgen ${ }^{20}$.

$\mathrm{Zu}$ erwähnen ist allerdings auch, dass diese Indizien keineswegs gefeit sind gegen falsch positive oder falsch negative Befunde. Sie werden deshalb selbstverständlich an Bedeutung für eine Gefährdungseinstufung verlieren, wenn zuverlässigere (z. B. toxikologische) Daten zur Verfügung stehen. Zudem wurde von VertreterInnen von Unternehmen in der Kommission die durchaus berechtigte Sorge formuliert, dass ein zunächst vorläufig als besorgniserregend eingestuftes Material allein dadurch für immer in der Öffentlichkeit „verbrannt" sei. Eine nachträgliche Entwarnung aufgrund valider toxikologischer Daten würde dann womöglich gar nichts mehr nützen.

\section{Abb. 2: Entlastungs- und Besorgniskriterien der NanoKommission}

\section{Entlastungskriterien}

\section{Verlust der Nanoeigenschaften durch:}

- Gute Löslichkeit (in Wasser, in Körperflüssigkeiten,...), wenn dadurch die Nanoeigenschaften verloren gehen

- Schnelle Abbaubarkeit (biologisch, photokatalytisch,...) in nicht toxische Abbauprodukte

- Feste und dauerhafte Einbindung in Matrices (Stabilität der Matrix, Bindungsart, „End-of-Life“-Verhalten)

- Vorliegen fest gebundener Aggregate (produktionsbedingt)

- Agglomerationsverhalten: Bildung stabiler, großer Agglomerate, (z. B. Größe, Stabilität,...)

- Nanostrukturierte Modifikationen an Oberflächen und Nanostrukturen, die keine Partikel freisetzen und nicht reaktiv sind (z. B. Nanoporen, Lotuseffekt,...).

\section{Besorgniskriterien}

\section{Hinweise auf erwartbar hohe Exposition:}

- Produktionsmenge bzw. Einsatzmenge für den Anwendungsbereich (Expositionswahrscheinlichkeit)

- Hohe Mobilität in Nanoform

- in Organismen ( Alveolengängigkeit, Persistenz in Wasser, Fett und Körperflüssigkeiten, Durchgang durch Zellmembranen, Blut-Hirn-Schranke, Placenta, Betrachtung des Sonderfalls von drug delivery systems)

- in der Umwelt (Ferntransport, Persistenz in Wasser und Fett, Löslichkeit in Fett und Wasser, Bioverfügbarkeit, Staubigkeit)

- Mobilisierungspotenzial (Huckepack, Einschleusung, Sorption, Komplexbildung)

- Gezielte Freisetzung (z. B. Grundwassersanierung, Agraranwendungen, verbrauchernahe Anwendungen, Innenraumanwendungen,...)

- Persistenz der Nanoeigenschaften

- Bioakkumulation

\section{Hinweise auf evtl. problematische Wirkungen:}

- Hohe Reaktivität (katalytisch / chemisch / biologisch)

- Problematische Morphologie (stabile, lange Röhren oder Fasern, aspect ratio, Fullerene, Kristallstruktur, Porosität)

- Hinweise aufproblematische Wechselwirkungen (z. B. Huckepack)

- Hinweise auf problematische Transformationen (Alterung, Veränderungen der Oberflächeneigenschaften, Porosität) oder Metaboliten (z. B. Veränderungen oder Verlust des Coatings)

\section{Hinweise auf Probleme im Risikomanagement:}

- Schlechte Nachweisbarkeit

- Unklarer Verbleib

Quelle: NanoKommission 2008, S. 44 


\section{Fazit-Ausblick}

Indizien und Indikatoren, die uns in die Lage versetzen, auf mögliche Probleme aufmerksam zu werden, lange bevor diese auftreten, sind wichtig in der Technikfolgenabschätzung. Sie helfen uns früh im Innovationsprozess Schäden zu vermeiden bzw. zu minimieren, wenn das Wissen über Folgen zwar gering, die Handlungsspielräume für Korrekturen aber noch groß sind. Sie ersparen uns die Mühen und Kosten der Reparatur und des Umsteuerns. Besonders wichtig sind Indizien und Indikatoren für Eingriffe und Technologien, die sehr tief in die Systeme eingreifen und dadurch so wirkmächtig werden, dass sie extrem weitreichende Konsequenzen nach sich ziehen können. Das Reparieren und Umsteuern ist dann kaum noch möglich (Besorgniskriterien: Eingriffstiefe und Wirkmächtigkeit). Beispiele hierfür sind die Freisetzung persistenter, mobiler und naturfremder synthetischer Chemikalien (wie die ,persistent organic pollutions" [POPs] und die FCKWs), die Produktion von radioaktiven Nukliden mit extrem langen Halbwertszeiten sowie die Freisetzungen von zur Selbstvermehrung fähigen gentechnisch veränderten oder synthetischen Organismen. Ähnliches gilt mit hoher Wahrscheinlichkeit auch für sehr mobile und persistente Nanomaterialien, die nach der Freisetzung ihre Nanoeigenschaften zu behalten in der Lage sind. In all diesen Fällen sollen allein schon die identifizierten Eigenschaften dazu führen, dass derartige Stoffe, Technologien und Objekte vor der Markteinführung einem spezifischen Prüfungs- und Zulassungsverfahren unterzogen werden müssen. Zudem soll die Pflicht zur Suche nach Alternativen oder Substituten vorgeschrieben werden. Und es müssen - wenn die Substitution nicht möglich sein sollte - schon sehr starke gesellschaftliche Nutzenaspekte ins Feld geführt werden, um den ursprünglich eingeschlagenen Entwicklungspfad trotz der großen Besorgnis weiter zu verfolgen.

\section{Anmerkungen}

1) Beim Versuch, sich einen Überblick über den Stand der bisherigen toxikologischen Erkenntnisse zu einem Stoff zu verschaffen, werden bestimmte Studien aus der Betrachtung ausge- schlossen. Dies geschieht mit der durchaus nachvollziehbaren und zu unterstützenden Begründung der Qualitätssicherung wissenschaftlicher Erkenntnis. Wichtig sind hier die „Prinzipien guter Laborpraxis" und der ,,weight of evidence approach" (vgl. OECD 1998; ECHA 2010 sowie zur aktuellen Debatte über Nanomaterialien Zuin et al. 2011). Ein solcher Ausschluss trifft allerdings auch Studien, die zwar noch keine nachweisbaren (evidenten) Dosis-Wirkungsbeziehungen oder Expositionswahrscheinlichkeiten begründen, aber immerhin schon Hinweise (Indizien) für einen „Anlass zur Besorgnis“ liefern.

2) Mit dem in REACH etablierten Grundprinzip „No data no market" wurde erfolgreich eine recht weitreichende Beweislastumkehr etabliert.

3) Hinter den so einfach erscheinenden Begriffen wie „Beweis“" und „Indiz“ bzw. „Indikator" stecken unzählige Definitions-, Begründungs- und Abgrenzungsprobleme, die hier nicht expliziert werden können. Schon innerhalb von wissenschaftlichen Disziplinen und erst recht im Vergleich zwischen ihnen zeigt sich ein breites Spektrum von disziplinär akzeptierten Evidenzgraden von Aussagen. Das zwischen sog. „Beweisen“ und „Indizien“ weitgespannte Spektrum ist somit am besten als Kontinuum darzustellen, ohne klare Grenzen zwischen beiden.

4) Ein solcher Besorgnisgrundsatz spielt auch im Wasser- und Bodenrecht eine wichtige Rolle (vgl. Calliess 2001).

5) Wobei hier noch einmal betont werden soll, dass auch die sog. Evidenzen mit zahlreichen Voraussetzungen und Unsicherheiten behaftet sind.

6) Collingridge (1980) hat schon früh auf die Zwickmühle zwischen geringem Wissen, aber weitreichenden Handlungsmöglichkeiten in der Frühphase von Innovationsprozessen und dem umgekehrten Fall in späteren Phasen des Innovierens hingewiesen und im Übrigen dafür plädiert, die Wissensanforderungen durch den Rückgriff auf robustere Technologien zu vermindern (vgl. Liebert/Schmidt 2012).

7) Vgl. immerhin die Arbeiten im und im Umkreis des SFB 536 „Reflexive Modernisierung“" sowie Wehling 2006; Böschen et al. 2010; Janich et al. 2012.

8) Weitere Beispiele finden sich in vielen nationalen, europäischen und internationalen Regelungen. $\mathrm{Zu}$ erwähnen ist hier z. B. die Novel-Food-Verordnung, in der sogar die bloße „Neuheit“" eines Stoffes oder einer Technik Vorsorgemaßnahmen auszulösen vermag.

9) Das Scheitern der so angelegten Chemikaliengesetzgebung der 1970er Jahre war schließlich ein 
ganz wesentlicher Grund für den umfassenden Neuanfang durch REACH (vgl. auch Choi et al. 2009).

10) Regulation (EC) No. 1907/2006 of the European Parliament and of the Council of 18 December 2006 concerning the Registration, Evaluation, Authorization and Restriction of Chemicals.

11) Vgl. Bruinen de Bruin et al. 2008; Vermeire et al. 2010.

12) Choi et al. 2009 schätzen die nötigen Aufwendungen für das Testen von Nanomaterialien im günstigsten Fall (NM erweisen sich im Wesentlichen als harmlos) auf 249 Mio. \$, im ungünstigsten Fall auf 1,18 Mrd. \$ (,for a more comprehensive precautionary approach“ (S. 3030)). „The time taken to complete testing is likely to be very high (34-53 years) if all existing nanomaterials are to be thoroughly tested“ (ibid.). Sie berechnen auch die Aufwände in Szenarien mit einem 1\%igen, 5\%igen und 10\%igen Anteil der Risikoforschung an der Gesamtförderung für ,,nanomaterial evaluation research" (ibid).

13) Vgl. Gleich et al. 2010.

14) Es bleibt zu erwähnen, dass mit dieser Regelung in REACH das Vorsorgeprinzip in einer Form rechtlich umgesetzt wird, die weit über die streckenweise schwachen Formulierungen in der „Kommunikation der Kommission“ zum Vorsorgeprinzip hinaus geht, vgl. Kommission der Europäischen Gemeinschaften 2000. Auf der anderen Seite muss, um REACH auch auf Nanomaterialien anwenden zu können, nicht nur die Stoffdefinition und die Tonnage-Regelung neu gefasst werden (Nanomaterialien werden eher in sehr kleinen Mengen eingesetzt), sondern auch die Gründe für große Besorgnis in Article 57 REACH (derzeit karzinogen, mutagen, reproduktionstoxisch, persistent, bioakkumulierbar und toxisch sowie sehr persistent und bioakkumulierbar) müssen um weitere nanospezifische Kriterien erweitert werden (vgl. Callies/Stockhaus 2012, S. 27ff.). Einen Ansatz dafür bieten die Besorgnis- und Entlastungskriterien der NanoKommission (2008).

15) Vgl. Weizsäcker/Weizsäcker 1984.

16) Technische Funktionalitäten beziehen sich auf das, was eine Technik (ein Eingriff) „,bewirken“ kann und zwar gleichermaßen im Sinne der technisch interessanten nutzbaren Potenziale (was sie also interessant macht) als auch im Sinne der Potenziale für adverse (unerwünschte) Neben- und Folgewirkungen.

17) Vgl. Gleich 1989, dort wird das technische Ansetzen an atomaren und molekularen Strukturen und an den Genen als Basis für hohe Wirkmächtigkeit hervorgehoben. Der Ansatz lässt sich aber auch auf das technische Ansetzen an Steuerungsstrukturen oder an ,systemrelevanten“ Strukturen erweitern, seien es Hormone, Nerven/Gehirn oder Bewusstsein in Organismen/Menschen oder elementare Stoff-, Energie- und Informationsflüsse in Ökosystemen.

18) Dass diese Blockade möglich war, beruhte auf mindestens drei Voraussetzungen: 1. eine Mindestresonanz in der (medialen) Öffentlichkeit für den von Umwelt-, Gesundheits- und Verbraucherschutzgruppen gespeisten Diskurs über etwaige Gefährdungen; 2. ein kaum erkennbarer Nutzen für die Endverbraucher (Nutzenclaims für gentechnische Verfahren in der Ernährungskette betrafen nur den Anbau, die Weiterverarbeitung und den Handel) sowie 3. reichlich gentechnikfreie Alternativen auf dem Markt.

19) Vgl. NanoKommission 2008 und 2011.

20) Als kleiner Lichtstreif am Horizont können immerhin die jüngst veröffentlichten Ergebnisse der NanoSafety Cluster Working Group 10 angesehen werden, die sich auf in vielen Bereichen identische Besorgniskriterien beziehen. Durch die dort vorgestellten Besorgniskriterien sollen allerdings keine Vorsorgemaßnahmen des Risikomanagements ausgelöst werden. Sie werden ausschließlich als Basis für eine besorgnisorientierte Gruppierung von Nanomaterialien verwendet und für die Fokussierung der weiter führenden Teststrategien (vgl. Oomen et al. 2013).

\section{Literatur}

Böschen, St.; Kastenhofer, K.; Rust, I. et al., 2010: The Political Dynamics of Scientific Non-Knowledge. In: Science, Technology \& Human Values 35/6 (2010), S. $783-811$

Bruinen de Bruin, Y.; Vermeire, Th.; van Engelen, J. et al., 2008: Exposure Informed Testing under REACH. In: Epidemiology 19 (2008), S. 199

Calliess, Chr., 2001: Vorsorgeprinzip und Beweislastverteilung im Verwaltungsrecht. In: DVB1. 2001, S. 1725-1733

Calliess, Chr.; Stockhaus, H., 2012: Precautionary Principle and Nanomaterials: REACH revisited. In: Journal for European Environmental \& Planning Law 9.2 (2012), S. 113-135

Choi, J.Y.; Ramachandran, G.; Kandlikar, M., 2009: The Impact of Toxicity Testing Costs on Nanomaterial Regulation. In: Environmental Science \& Technology 43/9 (2009), S. 3030-3034 
Collingridge, D., 1980: The Social Control of Technology. London

ECHA - European Chemicals Agency, 2010: Practical Guide 2: How to Report Weight of Evidence. Helsinki EEA - European Environment Agency, 2001: Late Lessons from Early Warnings: The Precautionary Principle 1896-2000. Environmental Issue Report No. 22/2001

EEA - European Environment Agency, 2013: Late Lessons from Early Warnings - Science, Precaution, Innovation. EEA Report No. 1/2013

Gleich, A. von, 1989: Der wissenschaftliche Umgang mit der Natur. Über die Vielfalt harter und sanfter Naturwissenschaften. Frankfurt a. M.

Gleich, A. von; Gößling-Reisemann, St.; Stührmann, S. et al., 2010: Resilienz als Leitkonzept - Vulnerabilität als analytische Kategorie. In: Fichter, K.; Gleich, A. von; Pfriem, R. et al. (Hg.): Theoretische Grundlagen für erfolgreiche Klimaanpassungsstrategien. nordwest2050-Berichte 1. Bremen, S. 11-49

Janich, N.; Nordmann, A.; Schebeck, L. (Hg.), 2012: Nichtwissenskommunikation in den Wissenschaften. Interdisziplinäre Zugänge. Frankfurt a. M. (Wissen Kompetenz - Text, Bd. 1), S. 235-267

Kommission der Europäischen Gemeinschaften, 2000: Mitteilung der Kommission - Die Anwendbarkeit des Vorsorgeprinzips. Brüssel; http://eur-lex.europa.eu/ LexUriServ/site/de/com/2000/com2000_0001de01. pdf (download 28.10.13)

Liebert, W.; Schmidt, J.C., 2012: Collingridge's Dilemma and Technoscience. In: Poiesis \& Praxis 7/1-2 (2012), S. 55-71

NanoKommission der Deutschen Bundesregierung, 2008: Verantwortlicher Umgang mit Nanotechnologien. Bericht und Empfehlungen der NanoKommission der Deutschen Bundesregierung 2008. Berlin; http:// www.bmu.de/files/pdfs/allgemein/application/pdf/ nanokomm_abschlussbericht_2008.pdf (download 28.10.13)

NanoKommission der Deutschen Bundesregierung, 2011: Verantwortlicher Umgang mit Nanotechnologien. Bericht und Empfehlungen der NanoKommission 2011. Berlin; http://www.bmu.de/files/pdfs/allgemein/application/pdf/nano_schlussbericht_2011_ bf.pdf (download 28.10.13)

Oomen, A.G.; Bos, P.M.; Fernandes, T.F. et al., 2013: Concern-driven Integrated Approaches to Nanomaterial Testing and Assessment - Report of the NanoSafety Cluster Working Group 10. Nanotoxicology
OECD - Organization for Economic Co-Operation and Development, 1998: Principles on Good Laboratory Practice (as revised in 1997). Paris

Vermeire, Th.; van den Bovenkamp, M.; Bruinen de Bruin, Y. et al., 2010: Exposure-based Waiving under REACH. In: Regulatory Toxicology and Pharmacology (RTP) 58/3 (2010), S. 408-420

Wehling, P., 2006: Im Schatten des Wissens? Perspektiven der Soziologie des Nichtwissens. Konstanz

Weizsäcker, Chr. von; Weizsäcker, E.U. von, 1984: Fehlerfreundlichkeit. In: Kornwachs, K. (Hg.): Offenheit Zeitlichkeit - Komplexität. Frankfurt a. M., S. 167-201

Zuin, St.; Micheletti, Chr.; Critto, A., 2011: Weight of Evidence Approach for the Relative Hazard Ranking of Nanomaterials. In: Nanotoxicology 5/3 (2011), S. $445-458$

\section{Kontakt}

Prof. Dr. Arnim von Gleich

Technikgestaltung und Technologieentwicklung

Fachbereich Produktionstechnik

Universität Bremen

Badgasteiner Straße 1, 28359 Bremen

Tel.: +4942164880

E-Mail: gleich@uni-bremen.de 